\title{
Detection of persistent sub-GeV $\gamma$-ray emission towards SS433/W50
}

Pol Bordas*

Max-Planck-Institut für Kernphysik, Heidelberg, Germany

E-mail: pol.bordas@mpi-hd.mpg.de

\section{Ruizhi Yang}

Max-Planck-Institut für Kernphysik, Heidelberg, Germany

E-mail: ryang@mpi-hd.mpg.de

\section{Ervin Kafexhiu}

Max-Planck-Institut für Kernphysik, Heidelberg, Germany

E-mail: ervin.kafexhiu@mpi-hd.mpg.de

\section{Felix Aharonian}

Max-Planck-Institut für Kernphysik, Heidelberg, Germany

School of Cosmic Physics, Dublin Institute for Advanced Studies, Ireland

E-mail: Felix.Aharonian@mpi-hd.mpg.de

\begin{abstract}
We report on the discovery of a persistent gamma-ray signal from a source positionally coincident with the microquasar SS433 and the surrounding W50 nebula. The gamma-ray flux is steady in the $\sim 5$ years of observations collected by the Fermi-LAT, and its spectral energy distribution displays a distinct maximum at $\sim 250 \mathrm{MeV}$, extending up to only $\sim 800 \mathrm{MeV}$. Given the large kinetic power and the known existence of baryonic material in the jets of SS433, we consider the possibility that the observed emission is produced through proton-proton collisions at the SS433/W50 interaction regions. Other scenarios cannot be ruled out, however, including gamma-ray emission produced by relativistic electrons through Inverse Compton or relativistic Bremsstrahlung processes in the jets of SS433, or the high-energy fluxes being originated in the W50 nebula itself. We refer to [1] for a more detailed discussion.
\end{abstract}

The 34th International Cosmic Ray Conference,

30 July- 6 August, 2015

The Hague, The Netherlands

\footnotetext{
* Speaker.
} 


\section{References}

[1] P. Bordas, R. Yang, E. Kafexhiu \& F. Aharonian, ApJ, 807L, 8B, 2015 